\title{
Could automated analysis of chest X-rays detect early bronchiectasis in children?
}

\author{
Alys R. Clark ${ }^{1}$ (1) Emily Jungmin $\mathrm{Her}^{2} \cdot$ Russell Metcalfe ${ }^{3} \cdot$ Catherine A. Byrnes $^{3,4}$ \\ Received: 1 November 2020 / Revised: 17 March 2021 / Accepted: 30 March 2021 / Published online: 28 April 2021 \\ (C) The Author(s), under exclusive licence to Springer-Verlag GmbH Germany, part of Springer Nature 2021
}

\begin{abstract} confidence level.

What is Known:

- Bronchiectasis is a severe chronic respiratory disorder increasingly recognised in paediatric populations.

- Diagnostic computed tomography imaging is often requested only after several chest $X$-ray investigations.

What is New:

- We show that a digital analysis of chest X-ray could provide more accurate identification of bronchiectasis features.
\end{abstract}

Non-cystic fibrosis bronchiectasis is increasingly described in the paediatric population. While diagnosis is by high-resolution chest computed tomography (CT), chest X-rays (CXRs) remain a first-line investigation. CXRs are currently insensitive in their detection of bronchiectasis. We aim to determine if quantitative digital analysis allows CT features of bronchiectasis to be detected in contemporaneously taken CXRs. Regions of radiologically (A) normal, (B) severe bronchiectasis, (C) mild airway dilation and (D) other parenchymal abnormalities were identified in CT and mapped to corresponding CXR. An artificial neural network (ANN) algorithm was used to characterise regions of classes A, B, C and D. The algorithm was then tested in 13 subjects and compared to CT scan features. Structural changes in CT were reflected in CXR, including mild airway dilation. The areas under the receiver operator curve for ANN feature detection were 0.74 (class A), 0.71 (class B), 0.76 (class C) and 0.86 (class D). CXR analysis identified CT measures of abnormality with a better correlation than standard radiological scoring at the $99 \%$

Conclusion: Regional abnormalities can be detected by digital analysis of CXR, which may provide a low-cost and readily available tool to indicate the need for diagnostic CT and for ongoing disease monitoring.

Keywords Bronchiectasis · Children · Chest X-rays · Computed tomography · Image analysis

Communicated by Piet Leroy

Alys R. Clark

alys.clark@auckland.ac.nz

Emily Jungmin Her

emily.her@research.uwa.edu.au

Russell Metcalfe

r.metcalfe@auckland.ac.nz

Catherine A. Byrnes

c.byrnes@auckland.ac.nz
1 Auckland Bioengineering Institute, The University of Auckland, Private Bag 92019, Auckland 1142, New Zealand

2 Department of Physics, University of Western Australia, Perth, Australia

3 Starship Children's Hospital, Auckland District Health Board, Auckland, New Zealand

4 Department of Paediatrics: Child and Youth Health, University of Auckland, Auckland, New Zealand 


\section{Introduction}

Non-cystic fibrosis bronchiectasis is a progressive condition characterised by abnormal bronchial dilation, associated with frequent bacterial infections and inflammatory airway damage. Chest computed tomography (CT) is the gold standard for diagnosis and monitoring $[1,2]$. Bronchiectasis is an increasingly recognised problem, especially in populations where access to a diagnostic CT is not possible or practicable [3]. Since 2000, in New Zealand, hospitalisation rates for bronchiectasis overall have increased by $45 \%$ and mortality by $88 \%$ [4]. At diagnosis children already have severe disease, with $81 \%$ bilateral and $64 \%$ with 3 or more lobes involved [5], suggesting delayed referral. All children have had a prior chest X-ray (CXR) and some have had many. The sensitivity of CXR for bronchiectasis is low at $37 \%$ with specificity of $95 \%$ [6]. However, strong correlations between CXR and CT scoring of bronchiectasis have been reported [7, 8]. CXR has been shown to be more sensitive to early lung disease than pulmonary function testing [8], and persistent parenchymal densities in CXRs have been related to the eventual development of bronchiectasis $[9,10]$.

Modern CXRs are obtained digitally so can be analysed on pixel-by-pixel basis. Digital analyses of CXR have been developed to aid diagnosis of lung cancer, tuberculosis, covid-19 and other lung diseases but have focused on adult imaging [11-14]. These methods could potentially aid radiological interpretation of CXRs as they can highlight possibly abnormal lung parenchymal features. For example, regions of the lung that appear more or less homogeneous than expected or have certain textural properties (e.g. lines/circles). Just a small number of studies have digitally assessed CXRs in paediatric populations $[15,16]$, focusing on pneumonia. Digital assessment of CXR has performed as well in detecting paediatric pneumonia as similar analyses in an adult population $[16,17]$, indicating that digital analysis of paediatric CXRs is feasible. Such analysis of CXRs in those at risk of bronchiectasis could provide low-cost screening to suggest further investigation.

The aim of this study was to develop a proof-of-concept digital analysis of CXR imaging informed by a same day standardised chest CT. We hypothesised that this automated analysis could detect bronchiectasis in CXR. Ultimately this capability could lead to earlier referral, prior to chronic symptoms developing, for the definitive CT diagnosis in appropriate children. To reduce heterogeneity (including differing ages, etiologies and CT protocols), we elected to develop and test the analysis in a well-characterised cohort of 5-yearold children with mild to moderate bronchiectasis due to cystic fibrosis $(\mathrm{CF})$ where a single radiological protocol had been used [18].

\section{Methods}

\section{Subjects}

Twenty-four children with CF were enrolled from diagnosis after newborn screening to final study outcomes at 5 years of age in New Zealand. These were a subset of the 'Australasian Cystic Fibrosis Bronchoalveolar Trial (ACFBAL)' which explored the effectiveness of bronchoalveolar lavage in directing treatment of respiratory infection in young children with CF [18]. The 24 subjects had mean body mass index of 15.5 (range 12.6-17.6) and all but one had pancreatic insufficiency. Positive cultures for the final BAL/ever grown in respiratory cultures included 63\%/100\% Staphylococcus aureus, 50\%/ 96\% Haemophilus influenza, 17\%/71\% Streptococcus pneumoniae, $17 \% / 46 \%$ Moraxella catarrhalis, $8 \% / 29 \%$ Pseudomonas aeruginosa, 4\%/13\% Stenotrophomonas maltophilia and $38 \% / 75 \%$ Aspergillus fumigatus. The subjects had treatment for infection using standard study protocols [18], and courses of antibiotics were prospectively recorded with the participants having a mean 9.6 days (range 0-38 days) intravenous therapy prior to the final chest CT.

Pulmonary function was recorded by standard spirometry using American Thoracic Society criteria with percent predicted and $z$-scores calculated from British reference values [18]. Satisfactory results were available for $21 / 24$ subjects (the remaining 3 were excluded from analysis for pulmonary function but not radiological scoring). Mean forced expiratory volume in $1 \mathrm{~s}$ (FEV1) was $92.5 \%$ predicted (range $67-126 \%$ ) and mean forced vital capacity (FVC) was 96\% (range 66-136\%).

\section{Radiology and scoring}

The ACFBAL outcomes included a CXR and chest CT conducted at a time of health stability, as described previously [18]. All children were 5 years old at the time of imaging. In brief, a low-dose high-resolution CT with 1-mm collimation scans at intervals of $10 \mathrm{~mm}, 120 \mathrm{kVp}, 50 \mathrm{~mA}, 1 \mathrm{~s}$ from apex to base was obtained using a Siemens Sensation 64 machine (Siemens, Forchheim, Germany) and reconstructed using the b70 kernel. All underwent general anaesthesia with lungs inflated to $25 \mathrm{~cm} \mathrm{H}_{2} \mathrm{O}$ for inspiratory scans. All scans were of good diagnostic quality. An erect posteroanterior and lateral CXR was taken on the same day. The time between CT and CXR was not recorded, but as all were in the same day, they were acquired $<24 \mathrm{~h}$ apart. CT and CXRs were matched but de-identified. The $\mathrm{CT}$ was scored independently in the original ACFBAL study at the Erasmus Medical Centre, Rotterdam, The Netherlands (see acknowledgements) using the modified Brody-II CF chest CT score [19]. Brody-II provides a total score of up to 234 , comprising $0-72$ for bronchiectasis, 0-54 for airway wall thickening, 0-36 for mucous plugging, 0-54 for parenchymal disease and 0-18 for air 
trapping. CXRs were scored by a paediatric radiologist at Starship Children's Hospital, Auckland, New Zealand using the Brasfield score $[20,21]$ which starts with a total of 25 and deducts 0-4 each for air trapping, linear markings and nodular cystic lesions and $0-5$ each for general severity and large lesions.

\section{Global assessment of CT features and mapping to CXR}

Features of bronchiectasis were extracted from CT, (1) to provide non-subjective classification of disease status and (2) to isolate regions within the same subject's CXR that correspond with each feature. The lung field was segmented from both inspiratory CT and CXR using semi-automated algorithms (Supplementary Material A). Airways in CT were individually measured to determine whether bronchiectasis was present, as defined previously [22]. Airway lumen diameter (LD), airway outer diameter (AOD) and artery (vessel) diameter (VD) were measured along their shortest axis using an electronic calliper tool. Before measurement, images were enlarged to $400 \%$ zoom and the window width and level set to 1500 and -450 Hounsfield units (HU), respectively. Airway wall thickness $(\mathrm{AWT})$ was calculated using $\mathrm{AWT}=(\mathrm{AOD}-\mathrm{LD}) / 2$. The mean ratios of airway lumen to artery diameter (LD/VD) and airway wall thickness to arterial diameter (AWT/VD) were also calculated. All clearly visible airway and blood vessel pairs passing perpendicularly to the imaging plane (with a circular appearance) and with airway lumen diameter $>$ $0.5 \mathrm{~mm}$ were measured. An individual airway was considered to be bronchiectatic if $\mathrm{LD} / \mathrm{VD}>1$. As variation in chest $\mathrm{CT}$ pixel intensity at the whole lung scale has been shown to reflect parenchymal changes reflective of disease [23], global metrics (mean, standard deviation and coefficient of variation of pixel intensities) were calculated within the lung region in both CT and CXR.

In each set of CT images, regions of the lung were manually classified using OsiriX software (http://www. osirix-viewer.com/). Regions were divided into 4 types: (A) radiologically normal, (B) definitive bronchiectasis features, (C) dilated airway and blood vessel pairs, and/ or mild airway wall thickening, but not meeting criteria for bronchiectasis using the methods of Long et al. [22], and (D) 'other' parenchymal abnormalities. A region was classified as (C) if it contained a single dilated airway (defined by LD/VD), but was only classified as (B) if multiple airways were present meeting bronchiectatic dilation, or a region comprising multiple airways be segmented from CT where bronchiectasis was present throughout. A 3D map of each CT image with regions of structural abnormality identified was generated to use as a gold standard and each feature was mapped to a PA projection (Fig. 1a and b) to identify the same regions in each individual's CXR.

\section{Feature detection in CXR}

Following segmentation (Fig. 1c), the CXR lung field was split into regularly sized blocks of pixels for classification (Fig. 1d). Block sizes of $8 \times 8,15 \times 15$ and $20 \times 20$ pixels were assessed, to determine an optimal pixel block size for feature detection. Each pixel block was classified as A, B, C or D depending on comparison with CT (Fig. 1e). If more than $40 \%$ of the block was classified as B, C or D the whole block was classified to that category. Otherwise, as the area could not be classified as normal but contained only a small proportion of abnormal tissue, it was excluded. Excluded blocks equated to less than $0.05 \%$ of the total being neglected (total number of blocks within the lung region is 2500-3000 blocks for each subject). For each pixel block, 39 textural features were computed. These features were chosen based on a similar study which aimed to detect lung fibrosis in CT [24] and include histogram statistics, co-occurrence matrix features, run-length matrix features and a Gabor filter bank (Supplementary Material B). An artificial neural network (ANN) analysis was conducted (Supplementary Material B), to determine the optimal set of image features and block size. A training set of 11 subjects was chosen at random, providing at $20 \times 20$ pixel block resolution a total of 14,546 pixel blocks to train the ANN. The remaining 13 subjects were used as a test cohort with the CXRs mapped first and checked with the CT findings as the gold standard second.

\section{Statistics}

Clinical characteristics, radiology scores and pulmonary function tests are expressed as means and standard deviations when normally distributed (ascertained by KolmogorovSmirnov test) and medians and ranges when not. To compare means, the Student's $t$-test was used when distributions were normal and the Wilcoxon rank sum test when not. A Pearson correlation analysis was conducted to assess linear correlations between variables. When more than two measures were compared, or for comparison between multiple observations of the same measure, analysis of variance (ANOVA) was employed. A level of $p=0.05$ was used to indicate statistical significance. Statistical analysis was conducted using IBM SPSS software (SPSS Inc., Chicago, USA).

\section{Ethical approval}

Ethical approval for this study was obtained from the New Zealand Northern X Ethics Committee (NTX/12/EXP/018) and caregiver consent obtained. 


\section{HRCT identified features}

a
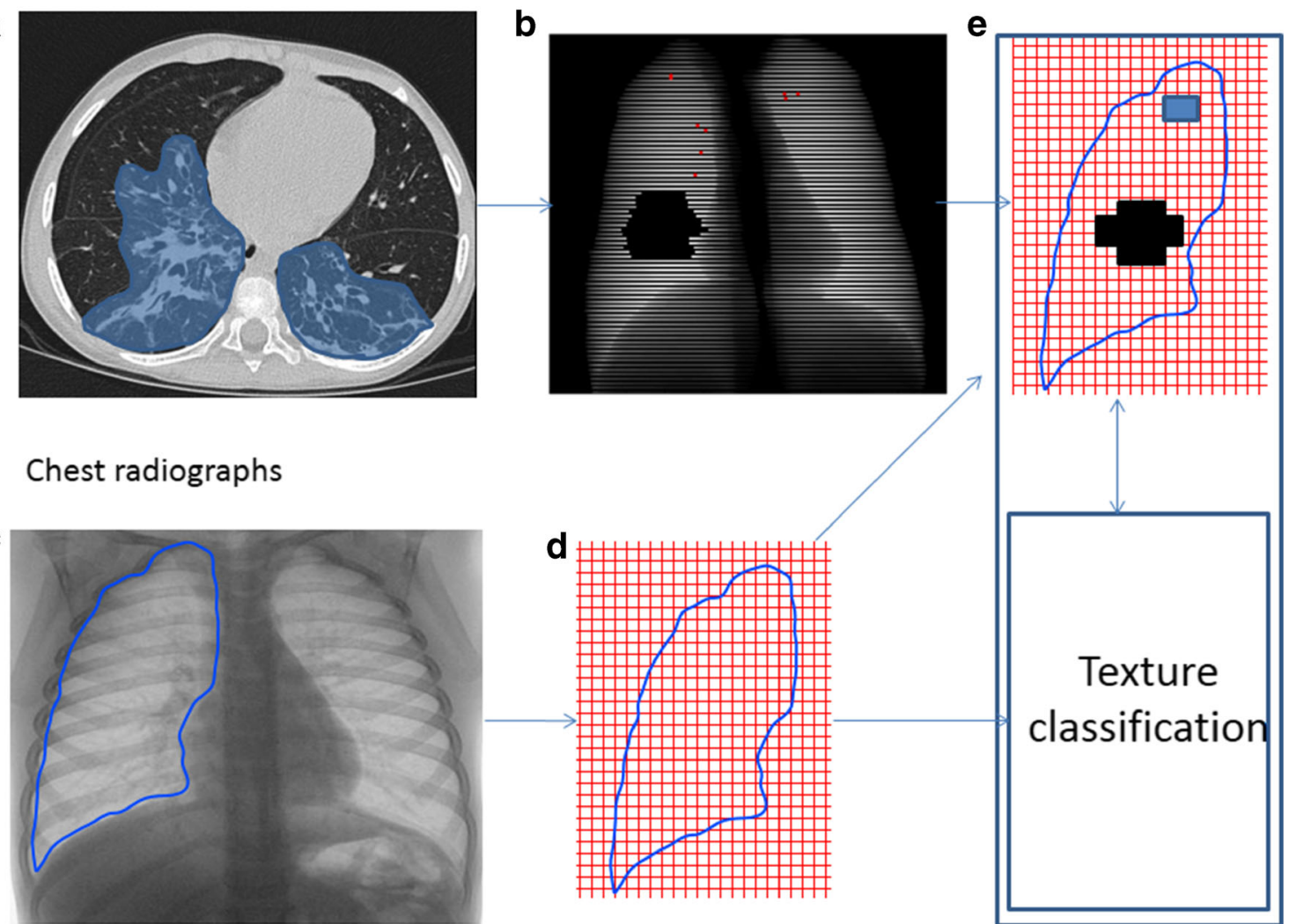

\section{Chest radiographs}

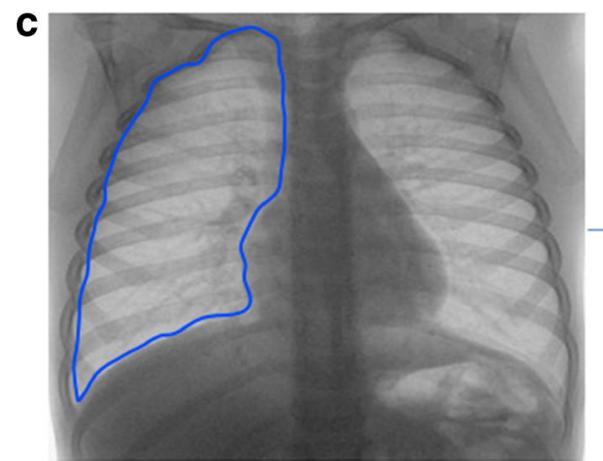

Fig. 1 Methodological steps for identifying regions of CXR for classification. a Abnormal regions are identified on CT and $\mathbf{b}$ mapped to the posteroanterior projection. $\mathbf{c}$ The lung shape is segmented from CXR images and $\mathbf{d}$ split into a grid of evenly sized squares. e Each

\section{Results}

\section{Radiological scoring and assessment of CT and CXR}

The CT (Brody-II) scores for this cohort were $3.2 \pm 2.7 \%$ (range $0-12.3 \%$ ) indicating mild lung disease. The component scores for bronchiectasis were $4.7 \pm 5.4 \%$ (range 0 $26.0 \%$ ), airway wall thickening $0.6 \pm 1.3 \%$ (range 0 $5.6 \%$ ), mucous plugging $2.6 \pm 3.3 \%$ (range $0-11.1 \%$ ), parenchymal abnormalities $3.7 \pm 2.9 \%$ (range $0-9.3 \%$ ) and air trapping $3.2 \pm 2.7 \%$ (range $0-50.0 \%$ ). Assessment of imaging in this cohort has been performed previously [18], and a brief summary of relationships between radiological scoring and pulmonary function is provided as Supplementary Material D.

\section{Global assessment of CT and CXR features}

The overall mean ratio of airway lumen to vessel diameter, $\mathrm{LD} / \mathrm{VD}$, measured directly from $\mathrm{CT}$ was $0.9 \pm 0.1$. This was slightly elevated but not significantly different $(p=$ 0.23 ) compared with the same measurements in healthy 5 -year olds from a previous study [22], further indicating square is classified by comparison to the posteroanterior CT projection, and classification of normal and abnormal regions conducted by an artificial neural network analysis

mild lung disease in this cohort. LD/VD correlated with the bronchiectasis component of the Brody-II score $(r=0.92$, $p=0.005$ ) and with CXR (Brasfield) scores $(r=-0.47, p=$ $0.01)$. LD/VD correlated with the prior number of days of IV therapy ( $r=0.48, p=0.02$ ) but again not with lung function (FEV1\% predicted $r=-0.32, p=0.17$ or FVC\% predicted $r=-0.35, p=0.17$ ). The airway wall thickness to vessel diameter ratio, AWT/VD, correlated with the total Brody-II score ( $r=0.44, p=0.03$ ) but, as in other studies $[22,25]$, had no significant relationship with other measures of lung health.

Mean lung density was $-797 \mathrm{HU}$, comparable to a previous estimate for inspiratory lung density in children of this age [26]. CT density coefficient of variation (defined as the ratio of standard deviation to the mean) correlated with both FEV1\% predicted $(r=0.45, p=0.05)$ and FVC\% predicted $(r=0.66, p=0.002)$. These relationships imply that reduced heterogeneity (for example areas of air trapping, consolidation) was linked to a reduced lung function. The coefficient of variation calculated from CXRs is correlated with the corresponding CT measure $(r=0.47, p$ $=0.05)$ but CXR heterogeneity was not sensitive enough to reflect changes in lung function. 
Fig. 2 Receiver operating characteristic (ROC) for detection of each class of tissue in CXR. The areas under the ROC curves are 0.74 (A-normal tissue), 0.71 (B - definitive bronchiectasis), 0.76 (C-airway dilation alone) and $0.86(\mathrm{D}$ - parenchymal abnormalities). True positives are blocks of tissue correctly classified by the algorithm and false positives are those identified in any incorrect class (e.g. a definitive bronchiectasis region identified as normal, airway dilation or parenchymal abnormalities)

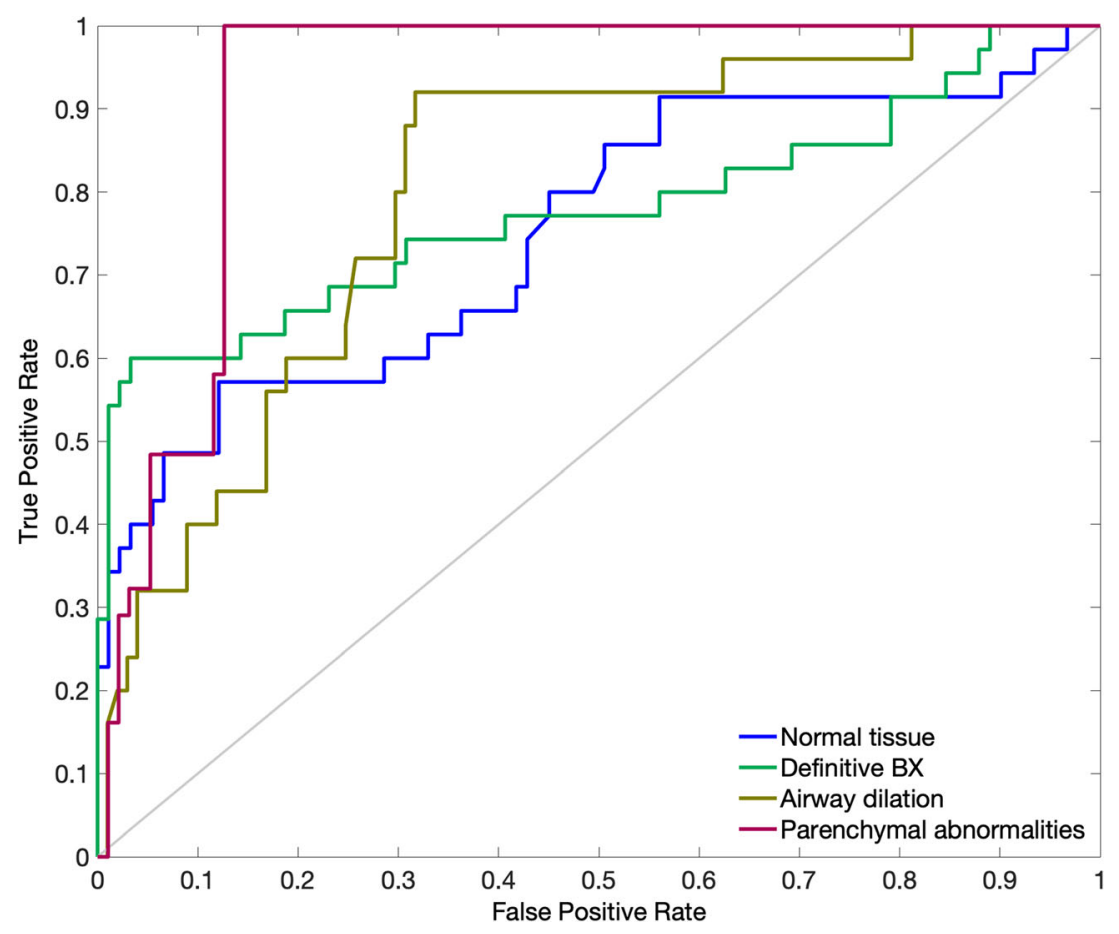

\section{Feature detection in CXR}

Preliminary analysis of the textural features in CXRs allowed reduction from 39 to 6 features to classify each image (Supplementary Material B). The optimal classification for the 11 test subjects was determined by the use of $20 \times 20$ pixel blocks for analysis and by including 500 neurons in the ANN. The receiver operating characteristic (ROC) is shown in Fig. 2 with areas under the ROC curve of 0.74 (A-normal tissue), 0.71 (B-definitive bronchiectasis), 0.76 (C-airway dilation alone) and $0.86(\mathrm{D}$ - other parenchymal abnormalities). In the 13 test subjects, the CXRs were classified and the percentage of tissue classified as abnormal was determined. Table 1 shows sensitivity and specificity for each BX feature identified indicating the performance of classification of each block in CXR. Specificity was greater than $80 \%$ in all cases. The sensitivities for the three lung abnormalities that were

Table 1 Sensitivity and specificity of the automated algorithm in identifying each of the bronchiectasis features analysed. As the most common misidentification ( $34 \%$ of all blocks) was between physiologically similar features, we also provide a sensitivity and specificity for identification as bronchiectasis ( $\mathrm{B}$ and $\mathrm{C}$ combined)

\begin{tabular}{lll}
\hline Abnormality & Sensitivity & Specificity \\
\hline Definitive bronchiectasis (B) & $60 \%$ & $92 \%$ \\
Airway dilation (C) & $56 \%$ & $84 \%$ \\
Parenchymal abnormalities (D) & $100 \%$ & $85 \%$ \\
Combined bronchiectasis features (B and C) & $77 \%$ & $81 \%$ \\
\hline
\end{tabular}

looked for ranged from 56 to $100 \%$, with identification of parenchymal abnormalities having the highest sensitivity. The lowest sensitivities were due to misidentification of definitive bronchiectasis (B) as dilated airways (C) and vice versa (34\% of all misidentified blocks). These features are morphologically similar, and the sensitivity of the algorithm in identifying bronchiectasis ( $\mathrm{B}$ and $\mathrm{C}$ combined) is calculated to be $77 \%$. Our automatically generated percentage of abnormal tissue in each CXR had greater correlation with CT measures of abnormality than radiologist scoring of CXRs in these test subjects (Fig. 3). Using the methods of Zou [27] and comparing Brasfield scoring and our method as dependent correlations, we confirmed that our method provided better correlation with AWT/LD, LD/VD and the total Brody score at the $99 \%$ confidence level.

\section{Discussion}

We have conducted a proof-of-concept quantitative assessment of chest CT and CXRs to identify early-stage bronchiectasis in CXR. Chest CT scans are well documented to be more sensitive to early structural changes than CXRs and remain the gold standard for diagnosing bronchiectasis and other parenchymal changes in both the CF and non-CF bronchiectasis populations $[1,2]$. However, CXRs are the more frequent and earlier investigation, are often repeated to determine resolution or persistence of early lung disease, and are more accessible to the community at large. A CT scan can be unaffordable and impractical in remote communities who 
Fig. 3 a, c, e Correlations between CT features of bronchiectasis and the number of pixel blocks detected by our automated algorithm as abnormal in 13 unseen subjects, and $\mathbf{b}, \mathbf{d}, \mathbf{f}$ the same CT features correlated with Brasfield scores in the same 13 subjects. In each case, the correlation is more significant using the automated analysis, than the semi-quantitative radiological assessment
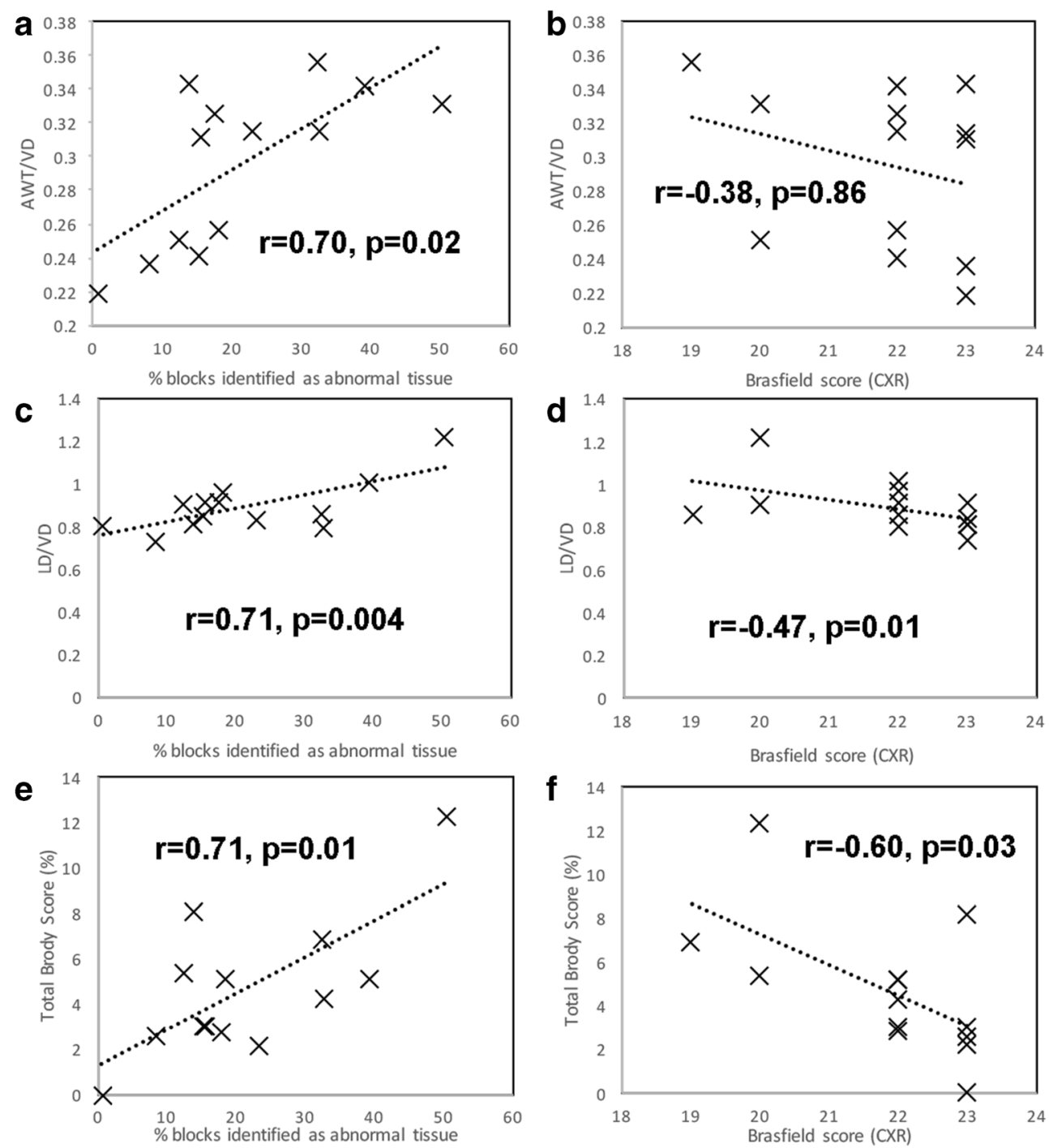

nonetheless have a high prevalence of chronic suppurative lung diseases including bronchiectasis [3]. Even in our setting, by time of bronchiectasis diagnosis children had chronic respiratory symptoms for a median of 38 months (2.9-7 years in 3 studies), $75 \%$ had hospital admissions at a median age of 1 year, with diagnosis at median age of 5.2 years and typically already had a number of CXRs [5, 28, 29]. While repeated low-dose chest CT is emerging for regular monitoring [30] and as an intervention trial outcome [31], increasing life expectancy in children with $\mathrm{CF}$ and bronchiectasis may temper the feasible frequency of this investigation [32]. Improving CXR sensitivity to predict the presence of significant structural damage - notably bronchiectasis - would improve the utility for screening, encouraging early referral and potentially have a role in more accurate disease monitoring over time.

We mapped areas of interest on the CT scan in the training set (half of the cohort) into four types - 'normal', 'bronchiectasis', 'dilated airway/bronchial wall thickening' or 'other' parenchymal abnormalities and converted this into the equivalent CXR. Specific airway and artery lumen and diameter measurements for all visible airway and artery pairs ensured accuracy of bronchiectasis assessment in CT. We were able to determine 6 features that could be assessed in small blocks of CXR images to identify each type of classified tissue. The CXR analysis in our test cohort showed ROC curves ranging $0.71-0.86$ for the 4 types of parenchymal appearance when compared to chest CT. The correlation between this analysis and quantitative CT measures of severity was superior to the correlation of traditional CXR scoring methods. Sensitivity of the algorithm to definitive bronchiectasis, airway dilation and parenchymal abnormalities ranges from 56 to $100 \%$, with $77 \%$ sensitivity when considering the algorithms ability to detect bronchiectasis (B and $\mathrm{C}$ combined). All calculated sensitivities are higher than the $37 \%$ previously reported in CXR [6], and calculated sensitivities and specificities are comparable to other automated algorithms that aim to detect disease features in CXR in other applications (typical ranges sensitivity $56-97 \%$, specificity $36-95 \%$ ) [33]. There 
is, in general, a trade-off between sensitivity and specificity in algorithms of this type. Future improvements to this algorithm to suppress unwanted features in CXR such as the ribs may enable improved sensitivity in the future, while maintaining comparable specificity.

The LD/VD ratios calculated here, representing the extent of airway dilation, correlated strongly with Brody II score. This is consistent with a previous study in more severe disease, which found a strong relationship between the two measures [34]. The LD/VD, the bronchiectasis component of the Brody-II score, and Brasfield scores all correlated strongly with the number of days of IV therapy suggesting a relationship to a clinical marker of disease severity in this cohort. Relationships between pulmonary function and CT scoring only become apparent when significant structural changes have already occurred [5, 34]. In this cohort, lower lung function relates to decreased heterogeneity in lung tissue that could arise from large regions of air trapping or consolidation, suggesting these changes, rather than emerging bronchiectasis (low-level airway dilatation) drive early pulmonary function loss.

Our cohort was carefully selected to eliminate possible confounders. Bronchiectasis is the main structural damage underlying CF lung disease $[1,25]$. We chose a young population likely to have early disease only, who had undergone a standardised protocol chest CT scan at a single age of 5 years imaged at a single centre. Previous studies have shown contradictory findings regarding correlation between age and airway dilation in children older than 2 years [25], perhaps due to different methods of airway sampling or centre effects. A nonCF bronchiectasis cohort was likely to be of different ages, has undergone varied protocols for CT scan acquisition and often already has severe disease at diagnosis. Larger prospective testing of these feature detecting algorithms in a more diverse dataset would provide important next steps toward a tool for bronchiectasis detection by CXR.

We have not addressed whether this type of CXR analysis for the presence of bronchiectasis provides an advantage over careful clinical assessment for a chronic wet cough. However, we already have evidence that children have prolonged cough for many years before progressing to the diagnostic investigation (chest CT) and instigation of correct management [5, 28, 29]. Shortening this delay, especially by using a cheap option such as CXR analysis, would be beneficial. Furthermore, the variability of CXRs is likely to be greater than CTs due to a wider range of equipment in a greater number of community and hospital settings. CXRs are also inherently noisy with ribs superimposed over the lung structure and the cardiac region was neglected in this study given the heart's dense opacity influence on the texture of lung tissue in this region. This means that bronchiectasis in the cardiac region would be missed using the technique as applied there. Techniques to account for variability in image protocols and other difficulties in computationally assessing CXRs have been discussed in detail [35], and a key improvement may be the implementation of rib suppression algorithms or algorithms that account for the presence of the heart in imaging. Finally, one study has described that the airway as being significantly smaller than the adjoining vessel in the younger paediatric age group suggesting that the current criteria derived from adult studies may already underestimate the presence of bronchiectasis [36].

Our proposed methodology improves upon standard radiological assessment of CXRs for bronchiectasis, providing preliminary evidence that digital analysis of CXR is feasible as a tool to screen for potential bronchiectasis early in disease progression and in community settings. We note that for widespread use, particularly in primary care, some additional testing of the algorithms may be necessary. For example, all CXRs and chest CT scans considered here were acquired in a single hospital setting, on the same machines, as is common in many digital analyses of similar imaging [11-14]. There may be variations in resolution and quality of CXR between sites that would need to be corrected for. Integrating assessment algorithms with CXR systems also presents a challenge; however, 'telehealth' or offsite interpretation has become commonplace in the pandemic experience of covid-19 and is more widely used now than previously, so this would not be seen as a significant hurdle.

In conclusion, we believe automated analysis of digital CXR in children is possible and can be used to improve the sensitivity to detect early bronchiectasis disease. A chest CT scan remains the gold standard for diagnosis of bronchiectasis but CXRs will continue to play a role, being more accessible and more frequently used. This automated assessment of CXR provides a means to improve the outcome of a test that is done frequently and is freely available to most communities. Enhancing interpretation to evolving chronic lung disease using simple measures of CXR textures will improve their utility encouraging early referral for definitive investigations and enhance their use for more accurate monitoring over time.

Abbreviations ACFBAL, Australasian Cystic Fibrosis Bronchoalveolar Lavage study; ANN, Artificial neural network; AOD, Airway outer diameter; AWD, Airway wall diameter; CF, Cystic fibrosis; CT, Computed tomography; CXR, Chest X-ray; FEV1, Forced expiratory volume in $1 \mathrm{~s}$; FVC, Forced vital capacity; LD, Airway lumen diameter; VD, Vessel diameter

Supplementary Information The online version contains supplementary material available at https://doi.org/10.1007/s00431-021-04061-8.

Acknowledgements We acknowledge the data provided by the Australasian Cystic Fibrosis Bronchoalveolar Lavage (ACFBAL) study group led by Professor Clair Wainwright (trial registration anzctr.org.au, identifier ACTRN12605000665639). The authors would like to thank Professor Harm Tiddens for supervising radiological scoring of HRCT as part of the ACFBAL study. 
Authors' contributions ARC conceived the study, funded the study, segmented images, compiled analysis of results, wrote the initial draft of the manuscript and edited and approved the manuscript. EJH wrote the code for the study, conducted ANN analysis, contributed to the initial draft of the manuscript and edited/approved the submission. RM scored X-ray images and edited/approved the manuscript. CAB conceived the study, compiled data for analysis and edited and approved the manuscript.

Funding This study was supported by a New Zealand Health Research Council Emerging Researcher First Grant (12/668).

Availability of data and material Data form part of the Australasian Cystic Fibrosis Bronchoalveolar Lavage (ACFBAL) research cohort, and any decisions regarding the use of the data or publications within the group and/or access to the data from outside the group must be discussed and agreed upon by a core panel designated by the ACFBAL researchers.

Code availability Code is available on reasonable request from the corresponding author.

\section{Declarations}

Ethics approval Ethical approval for this study was obtained from the New Zealand Northern X Ethics Committee (NTX/12/EXP/018).

Consent to participate Obtained.

Consent for publication Not applicable.

Conflict of interest The authors declare no competing interests.

\section{References}

1. Pasteur M, Bilton D, Hill A (2010) British Thoracic Society guideline for non-CF bronchiectasis. Thorax 65:11-i58

2. Chang AB, Bell SC, Torzillo PJ, King PT, Maguire GP, Byrnes CA, Holland AE, O’Mara P, Grimwood K, group ev (2015) Chronic suppurative lung disease and bronchiectasis in children and adults in Australia and New Zealand Thoracic Society of Australia and New Zealand guidelines. Med J Aust 202:130

3. Singleton R, Valery P, Morris P, Byrnes C, Grimwood K, Redding G, Torzillo P, McCallum G, Chikoyak L, Mobberly C, Holman R, Chang A (2014) Indigenous children from three countries with noncystic fibrosis chronic suppurative lung disease/bronchiectasis. Pediatr Pulmonol 49:189-200

4. Telfar B, Zhang J (2018) The impact of respiratory disease in New Zealand. 2018 update. Asthma and Respiratory Foundation of New Zealand, Wellington, New Zealand

5. Twiss J, Metcalfe R, Edwards E, Byrnes C (2005) New Zealand national incidence of bronchiectasis "too high" for a developed country. Arch Dis Child 90:737-740

6. Cooke J, Currie D, Morgan A, Kerr I, Delany D, Strickland B, Cole P (1987) Role of computed tomography in diagnosis of bronchiectasis. Thorax 42:272-277

7. van der Bruggen-Bogaarts $B$, van der Bruggen $H$, van Waes $P$, Lammers JW (1996) Screening for bronchiectasis: a comparative study between chest radiography and high-resolution CT. Chest 109:608-611
8. Sanders D, Li Z, Rock M, Brody A, Farrell P (2012) The sensitivity of lung disease surrogates in detecting chest CT abnormalities in children with cystic fibrosis. Pediatr Pulmonol 47:567-573

9. Redding G, Singleton R, Lewis T, Martinez P, Buttler J, Stamley D, Bulkow L, Peteres H, Gove J, Morray B, Jones C (2004) Early radiographic and clinical features associated with bronchiectasis in children. Pediatr Pulmonol 37:297-304

10. Valery P, Torzillo P, Mulholland K, Boyce N, Purdie D, Chang A (2004) Hospital-based case-control study of bronchiectasis in indigenous children in Central Australia. Pediatr Infect Dis J 23:902-908

11. Hoop V, de Boo D, Gietema H, van Hoorn F, Mearadji B, Schijf L, van Ginneken B, Prokop M, Schaefer-Prokop C (2010) Computeraided detection of lung cancer on chest radiographs: effect on observer performance. Radiology 257:532-540

12. Maduskar P, Muyoyeta M, Ayles H, Hogeweg L, Peters-Bax L, van Ginneken B (2013) Detection of tuberculosis using digital chest radiography: automated reading vs. interpretation by clinical officers. Int J Tuberc Lung Dis 17:1613-1620

13. Toussie D, Voutsinas N, Finkelstein M, Cedillo M, Manna S, Maron S, Jacobi A, Chung M, Bernheim A, Eber C, Concepcion J, Fayad Z, Gupta Y (2020) Clinical and chest radiography features determine patient outcomes in young and middle age adults with COVID-19. Radiology 297(1):E197-206

14. Qin C, Yao D, Shi Y, Song Z (2018) Computer-aided detection in chest radiography based on artificial intelligence: a survey. Biomed Eng Online 17:113

15. Mahomed N, van Ginneken B, Philipsen R, Melendez J, Moore D, Moodley H, Sewchuran T, Mathew D, Madhi S (2020) Computeraided diagnosis for World Health Organization-defined chest radiograph primary-endpoint pneumonia in children. Pediatr Radiol 50: 482-491

16. Mouton A, Pitcher R, Douglas T (2010) Computer-aided detection of pulmonary pathology in pediatric chest radiographs. Lect Notes Comput Sci 6363:619-625

17. Van Ginneken B, Katsuragawa S, Romeny B, Doi K, Viergever M (2002) Automatic detection of abnormalities in chest radiographs using local texture analysis. IEEE Trans Med Imaging 21:139-149

18. Wainwright C, Vidmar S, Armstrong D, Byrnes C, Carlin J, Cheney J, Cooper P, Grimwood K, Moodie M, Robertson C, Tiddens H (2011) Effect of bronchoalveolar lavage-directed therapy on Pseudomonas aeruginosa infection and structural lung injury in children with cystic fibrosis. JAMA 306:163-171

19. Brody A, Klein J, Molina P, Quan J, Bean J, Wilmott R (2004) High-resolution computed tomography in young patients with cystic fibrosis: distribution of abnormalities and correlation with pulmonary function tests. J Pediatr 145:32-38

20. Brasfield D, Hicks G, Soong S, Tiller R (1979) The chest roentgenogram in cystic fibrosis: a new scoring system. Pediatrics 63:24-29

21. Brasfield D, Hicks G, Soong S, Peters J, Tiller R (1980) Evaluation of scoring system of the chest radiograph in cystic fibrosis: a collaborative study. American Journal of Roentgenology. 134(6): 1195-8

22. Long F, Williams R, Castile R (2004) Structural airway abnormalities in infants and young children with cystic fibrosis. J Pediatr 144:154-161

23. Subramaniam K, Clark A, Hoffman E, Tawhai M (2018) Metrics of lung tissue heterogeneity depend on BMI but not age. J Appl Physiol 125:328-339

24. Caban J, Yao J, Avila N, Fontana J, Mananiello V (2007) Texturebased computer-aided diagnosis system for lung fibrosis. Proc SPIE 6514, medical imaging 2007: computer-aided diagnosis 651439:18

25. de Jong P, Lindblad A, Rubin L, Hop W, de Jongste J, Brink M, Tiddens H (2006) Progression of lung disease on computed tomography and pulmonary function tests in children and adults with cystic fibrosis. Thorax 61:80-85 
26. Long F, Williams R, Castile R (2005) Inspiratory and expiratory CT lung density in infants and young children. Pediatr Radiol 35:677683

27. Zou G (2007) Toward using confidence intervals to compare correlations. Psychol Methods 12:339-413

28. Edwards EA, Asher MI, Byrnes CA (2003) Paediatric bronchiectasis in the twenty-first century: experience of a tertiary children's hospital in New Zealand. J Paediatr Child Health 39:111-117

29. Munro K, Reed P, Joyce H, Perry D, Twiss J, Byrnes C, Edwards E (2011) Do New Zealand children with non-cystic fibrosis bronchiectasis show disease progression? Pediatr Pulmonol 46:131-138

30. Mott L, Park J, Murray C, Gangell C, de Klerk N, Robinson P, Robertson C, Ranganathan S, Sly P, Stick S, CF oboA (2012) Progression of early structural lung disease in young children with cystic fibrosis assessed using CT. Thorax 67:509-516

31. Davis S, Brody A, Emond M, Brumback L, Rosenfeld M (2007) Endpoints for clinical trials in young children with cystic fibrosis. Proc Am Thorac Soc 4:418-430

32. O'Connell $\mathrm{O}$, McWilliams S, McGarrigle A, O'Connor O, Shanahan F, Mullane D, Eustace J, Maher M, Plant B (2012)
Radiologic imaging in cystic fibrosis: cumulative effective dose and changing trends over 2 decades. Chest 141:1575-1583

33. Harris M, Qi A, Jeagal L, Torabi N, Menzies D, Korobitsyn A, Pai M, Nathavitharana R, Ahmad Khan F (2019) A systematic review of the diagnostic accuracy of artificial intelligence-based computer programs to analyze chest X-rays for pulmonary tuberculosis. PLoS One 14:e221339

34. Terheggen-Lagro S, Arets H, van der Laag J, van der Ent C (2007) Radiological and functional changes over 3 years in young children with cystic fibrosis. Eur Respir J 30:279-285

35. Van Ginneken B, Hogeweg L, Prokop M (2009) Computer-aided diagnosis in chest radiography: beyond nodules. Eur Respir J 72: 226-230

36. Kapur N, Masel J, Watson D, Masters I, Chang A (2011) Bronchoarterial ratio on high-resolution CT scan of the chest in children without pulmonary pathology. Chest 139:1445-1450

Publisher's note Springer Nature remains neutral with regard to jurisdictional claims in published maps and institutional affiliations. 